\title{
Disturbances in Metabolism of Phenylalanine and Tyrosine as an Important Factor in the Etiology and Pathogenesis of Psychoneurological Disorders Associated with Liver Diseases
}

\author{
Vadim P. Komovi ${ }^{1}$ Sergey E. Khalchitsky ${ }^{2}$; Michael V. Dubina ${ }^{3}$ \\ ${ }^{1}$ Saint Petersburg State Chemical Pharmaceutical Academy \\ ${ }^{2}$ Russian Research Center of Radiology and Surgical Technologies \\ ${ }^{3}$ Saint Petersburg Academic University \\ St. Petersburg, Russia
}

\begin{abstract}
Derangements of phenylalanine and tyrosine metabolism are an important factor in the etiology and pathogenesis of psychoneurological disorders. These disorders are particularly pronounced with monogenic hereditary diseases. In this work, we investigated similar disturbances in widespread and socially significant diseases - viral hepatitis and chronic alcoholism which are accompanied by liver damage. We found serious derangements in the metabolism of phenylalanine, tyrosine and their derivatives with these diseases and concluded that such derangements make an essential contribution to the development of psychoneurological disorders in the studied pathology. In conclusion, this paper proposes recommendations for correction of such derangements and normalization of the patients' condition.
\end{abstract}

Keywords: phenylalanine; tyrosine; liver pathology; psychoneurological disorders.

\section{Introduction}

Phenylalanine (Phe) and tyrosine (Tyr) are the aromatic amino acids (AAA) that play an important role in neurotransmitter biosynthesis. Normally the concentration of phenylalanine in blood does not exceed $2 \mathrm{mg} / 100 \mathrm{ml}$. When there are hereditary defects of the phenylalanine hydroxylase gene and other genes participating in Phe metabolism, there is a full or partial block of Phe transformation into tyrosine; thus, synthesized protein in whole or in part is not able to catalyze the transformation of Phe into Tyr. As a result, the concentration of Phe in the blood of patients with phenylketonuria (PKU)/ hyperphenylalaninemia (HPA) reaches up to 20 to 30 $\mathrm{mg} / 100 \mathrm{ml}$. These elevated concentrations lead to the activation of alternative ways of Phe metabolism and the formation of a number of products that are toxic to the organism, such as phenylpyruvic, phenyllactic, and phenylacetic acids.

*Corresponding author: Sergey E. Khalchitsky, PhD, Senior Research Scientist, Russian Research Center of Radiology and Surgical Technologies; St. Petersburg, Russia.

E-mail: s khalchitski@mail.ru
Toxic products of phenylalanine disintegration have a negative influence, primarily on the central nervous system (CNS). Patients with HPA have mental retardation, spasms, microcephaly, psychiatric disorders, etc. [1-4].

Except for hereditary disturbances of Phe metabolism, HPA often arises when various nonhereditary pathologies take place, in particular the diseases related to liver pathology. It is well known that the main enzyme of Phe biotransformation is phenylalanine hydroxylase (PAH) and that it is generally expressed in the liver; so any complicated diseases related to liver pathology can lead to disturbance of this enzyme functioning and, as a result, to HPA.

In the study of viral hepatitis (VH) and other liver damage, the special attention has been focused on the pathogenesis of hepatic encephalopathy (HE), the emergence mechanism which is still being discussed; but the majority of researchers do not consider the imbalance of AAA and nitrogen to be the main reason for HE emergence [5-7]. It should be noted that in $8 \%$ to $15 \%$ of cases, the reason for HE emergence, accompanying acute hepatic failure (AHF), remains obscure, and the general lethality is $80 \%$ among patients who do not receive a liver transplant [8]. This 
operation considerably reduces the intensity of HE symptoms (behavior and consciousness disorders, neuromuscular disorders etc.), ie, the pathology of metabolic processes in a liver is the trigger for HE emergence not only with $\mathrm{VH}$ but also with other hepatitides.

Disturbances of Phe, Tyr, and Trp metabolism are established in hereditary $[9,10]$ as well as in exogenous liver damages [8,11]. The activity of enzymes participating in Tyr and Trp transformations is modified, which distorts the metabolism of catecholamines $[12,13]$. Genetically determined activity of phenylalanine, tyrosine, tryptophan hydroxylases and their cofactor tetrhydrobiopterin, as well as other enzymes of these amino acids' metabolism, determines various forms of $\mathrm{VH}$ progress and the development of psychoneurological disorders with hepatic insufficiency, depending on the genetic constitution of each patient. Among the variety of biochemical disturbances with alcoholism, pathology of biogenic amine metabolism plays a special role. A special emphasis is put on the disturbance of the synthesis and metabolism of AAA (Phe, Tyr, and Trp). A change in the metabolism of these acids leads to an imbalance in the CNS neurotransmitters: adrenaline, noradrenaline, dopamine, and serotonin. Derangements in the metabolism of these neurotransmitters are considered by S. Mukherjee et al. [14] to be a cause of mental and neurological disorders with alcoholism.

Currently, no consensus has been reached concerning the mechanism of alcohol influence on the metabolism of catecholamines. The available data are devoted generally to the studies of Tyr and Trp metabolism. Meanwhile, studying the PAH-system of alcoholics' livers is extremely important for understanding the pathogenetic mechanisms that disturb the metabolism of biogenic amines, and such study provides valuable information about the causes of psychiatric and behavioral disorders, as well as neurological disorders, with alcoholism. About $90 \%$ of the ethanol a person consumes is metabolized in the liver [15], which causes harsh disorders of metabolic processes in this organ with chronic alcoholism (CA) [16]. In the livers of patients suffering from alcoholism, necrotic patches appear and chronic hepatitis and cirrhosis are developed [15]. Similar morphological disturbances found in patients with viral hepatitis are accompanied by a decrease in the activity of the liver PAH-system [17]. Possibly with CA, phenylalanine-4-hydroxylase (EC 1.14.3.1.) activity, as it is the main component of the liver PAH system, also decreases because the Phe concentration in the blood of alcoholics increases [18]. However, no study has yet been carried out about the condition of the liver PAH- system and the metabolism of phenylalanine with CA in the acute period of alcoholic intoxication with expressed mental disturbances. We observed patients with liver diseases, such as $\mathrm{VH}$ and $\mathrm{CA}$, in an effort to estimate Phe and Tyr metabolism and the influence of metabolic derangements on the development of psychoneurological disorders.

The aim of the this research was to find the severity of the Phe and Tyr metabolism disturbances and their influence on the genesis of psychoneurological disorders for patients suffering from $\mathrm{VH}$ and $\mathrm{CA}$ and to find out the dependence of these derangements on the severity of the pathological process.

\section{Material and Methods}

To analyze Phe and Tyr metabolism disturbances and to estimate the condition of the liver PAH-system at $\mathrm{VH}$, we studied 80 patients aged from 16 to 70 years with an acute form of hepatitis A and B. The diagnosis was made on the basis of the detection of viral hepatitis markers (anti-HAV IgM, anti-HAV $\mathrm{IgG}$ for hepatitis A and anti-HBs, anti-HBe and anti-HBc for hepatitis B) using enzyme immunoassay. The severity of the disease course was estimated from the degree of intoxication, a hemorrhagic syndrome, and by laboratory criteria and other clinical indicators. In particular, cases considered as a mild form of the disease were without intoxication, but with small jaundice, serum bilirubin $<85 \mu \mathrm{mol} / 1$, the prothrombin ratio (70-60\%) within norm, and increased activity of transaminases by 5 to 10 times.

Clinical cases considered as moderate-to-severe forms of disease included the obvious symptoms of intoxication, an expressed jaundice, bilirubinemia within 85 to $200 \mu \mathrm{mol} / 1$, a low prothrombin ratio, and increased activity of transaminases by 10 to 15 times. The severe form of the disease included vividly expressed intoxication, obvious jaundice, manifestations of a hemorrhagic syndrome, the phenomena of AHF and HE symptoms, prothrombin ratio $<60 \%$, neutrophilic leukocytosis, and hypoalbuminemia. Out of 80 adult patients, a severe form of $\mathrm{VH}$ was detected in 42 patients, a moderateto-severe form of $\mathrm{VH}$ in 28 patients, and a mild form of $\mathrm{VH}$ in 10 patients.

We determined the serum concentration of Phe, Tyr, epinephrine, norepinephrine, and serotonin, as well as the urinary excretion of phenylpyruvic acid (PPA), orthohydroxyphenylacetic acid (OHPAA), homogentisic acid (HGA), epinephrine, and norepinephrine. All patients underwent blood and urine tests at the peak of the disease, at the regression of clinical manifestations, and during recovery. The serum level of Phe was determined by the fluorimetric method [17] with using a 5•10-3 M solution of L-leucyl-Lalanine. The concentration of tyrosine was also determined by the fluorimetric method [17], and the level of PPA in urine was carried out according to I.A. Bulycheva et al. [19].

To estimate the activity of the liver PAH system, 16 patients (12 with a severe form and 4 with a moderate-tosevere form of $\mathrm{VH}$ ) were selected for oral administration of Phe in a dose of $0.1 \mathrm{~g} / \mathrm{kg}$. The control group consisted of 20 healthy people aged from 17 to 35 years. The peroral loading test was carried out on an empty stomach; the blood test was carried out before the loading and after 1, 2, 4 and 6 hours. Blood samples were taken from an elbow vein. Serum levels of Phe, Tyr, and catecholamines were determined. Excretion of PPA, OHPAA, HGA, epinephrine, and norepinephrine in daily urine were measured before, during, and after 24 hours of Phe load.

To estimate PAH activity, a direct determination of enzyme activity in the liver bioptates of $5 \mathrm{VH}$ patients was carried out. Bioptates (mass from 21 to $159 \mathrm{mg}$ ) were quickly processed; PAH activity was defined in supernatant liquid [17] using tetrahydrobiopterin (2-amino-4-hydroxy-6,7-dimethyltetrahydrobiopterin) (BH4) as a cofactor. The control group 
consisted of 11 people aged 25 to 30 years without liver pathology.

For identification of biochemical disturbances in the metabolism of Phe and Tyr, 34 CA patients, males aged between 25 and 48 years, were examined. Among these patients, 12 men were in their first day of hospitalization with the evident alcoholic abstinence syndrome; the remaining patients had undergone at least a 30-day treatment with complete abstinence from alcohol. The vast majority of patients had stage 2 alcoholism. The stages of alcoholism were estimated according to Goffman's classification [20]. The control group consisted of 20 healthy volunteers, males aged 21 to 35 years.

For identification of mutations in the PAH gene, $156 \mathrm{CA}$ patients (120 men and 36 women between 25 and 70 years) were examined. Alcoholism in stages 2 and 3, complicated with psychoneurological disorders and cases of delirium tremens, was marked in the anamnesis. The control group consisted of 417 volunteers of the same age.

DNA was extracted from peripheral blood leukocytes using "DNA-sorb" diagnostic sets produced by the DNAtechnology company (Russia) according to the manufacturer's protocol. The R408W mutation leading to a sharp decrease in PAH activity was analyzed by PCR/RFLP. A PKU-408 diagnostic set produced by the Center for Molecular Genetics (Russia) was used.

The study was conducted in accordance with ethical principles of the Declaration of Helsinki. It was approved by the local Ethics Committees. Written informed consent was obtained from all participants.

Results were statistically processed using the software package Statistica 6.0. Group comparisons with respect to categorical variables were performed using chi-square tests or, alternatively, Fisher's exact test when expected cell counts were less than 5 .

\section{Result and Discussion}

Table 1 shows the serum levels of Phe and Tyr as well as concentrations of PPA, epinephrine, and norepinephrine in the urine of $\mathrm{VH}$ patients. These data demonstrate an increase in serum Phe level, which is accompanied by increased urinary excretion of PPA. The increase of serum Phe concentration depends directly on the severity of the pathological process.
Table 2 shows the results of Phe load in $\mathrm{VH}$ patients with the severe form of disease at the peak of manifestation. The results of Phe load in the patients with $\mathrm{VH}$ and AHF are close to the changes observed in patients who are heterozygous for the R408W mutation, which is characterized by a decrease in PAH activity. Therefore, it is reasonable to assume that psychoneurological symptoms in VH patients, to a certain extent, may be caused by disturbances in Phe and Tyr metabolism and accumulation of toxic metabolites as a result of reduction in PAH activity (Table 3 ).

Phe load revealed a reduction in hepatic PAH activity in $\mathrm{VH}$ patients. The biochemical disturbances in Phe metabolism found with $\mathrm{VH}$ were also characteristic for PKU patients. Our data are consistent with the results received for Phe load in the heterozygous carriers of the R408W mutation, when depression of the PAH system is genetically caused [21].

Before Phe load, the elevated serum content of amino acids and catecholamines was associated with the severity of VH. After Phe load, the serum level of amino acid increased in all patients; 6 hours after loading, the serum level of this acid also exceeded the initial level. The increase in serum Tyr concentration was rather small. Urinary excretion of epinephrine and norepinephrine did not change significantly.

For the proof of disturbance of PAH activity in the liver, we determined the enzyme activity in liver bioptates of $5 \mathrm{VH}$ patients (Table 3) and found that the hepatic PAH activity was reduced by various degrees. PAH activity in the liver of patients with a mild form of $\mathrm{VH}$ at the peak of the disease and in the regression stage (patients \#1 and \#4; Table 3) was from 55.2 to $50.0 \mathrm{micromoles}$ of $\mathrm{Tyr} / \mathrm{g}$ protein/hour, respectively, and 18.8, 23.6,30.6 (mean 24.3 \pm 3.4 micromoles of Tyr/g protein/hour) (patients \#2, \#3 and \#5; Table 3) in patients with a moderate-to-severe form $\mathrm{VH}$ at the peak of disease and in the regression stage, respectively. The serum Phe level also increased in these patients. It is interesting to note that the enzyme activity was reduced more in patients with a high serum Phe level. Furthermore, the more severe form of the disease was accompanied by a pronounced decrease in PAH activity. Thus, the direct estimation of enzyme activity in the livers of patients with various clinical manifestations showed that the severity of disease is associated with the severity of disorders in the metabolism of Phe and Tyr.

Table 1. Concentration of Phe and Tyr in the blood serum; PPA, epinephrine and norepinephrine in daily urine of VH patients

\begin{tabular}{|c|c|c|c|c|c|c|}
\hline \multirow{2}{*}{ Group } & \multirow{2}{*}{$\begin{array}{l}\text { Stage of } \\
\text { disease }\end{array}$} & Phe & Tyr & PPA & Epinephrine & Norepinephrine \\
\hline & & \multicolumn{2}{|c|}{$(\mathrm{mg} / 100 \mathrm{ml})$} & $(\mathrm{mg})$ & \multicolumn{2}{|r|}{$(\mu \mathrm{g})$} \\
\hline \multirow{4}{*}{$\begin{array}{c}\text { Moderate-to-severe } \\
\text { form } \\
\text { of } \mathrm{VH}\end{array}$} & Peak of disease & $2.9 \pm 0.53$ & $1.8 \pm 0.18$ & $108.0 \pm 13.5$ & $7.8 \pm 0.70$ & $37.0 \pm 9.6$ \\
\hline & $P$ & $<0.01$ & $<0.01$ & $<0.01$ & $<0.01$ & $<0.4$ \\
\hline & Regression stage & $2.1 \pm 0.36$ & $1.7 \pm 0.20$ & $40.0 \pm 8.2$ & $6.5 \pm 0.34$ & $44.7 \pm 2.8$ \\
\hline & $P$ & $<0.04$ & $<0.01$ & $<0.01$ & $<0.05$ & $<0.01$ \\
\hline \multirow{4}{*}{$\begin{array}{c}\text { Severe } \\
\text { form } \\
\text { of } \mathrm{VH}\end{array}$} & Peak of disease & $4.5 \pm 0.55$ & $2.5 \pm 0.17$ & $160 \pm 15.6$ & $8.8 \pm 0.85$ & $48.2 \pm 12.8$ \\
\hline & $P$ & $<0.01$ & $<0.01$ & $<0.01$ & $<0.01$ & $<0.2$ \\
\hline & Regression stage & $2.3 \pm 0.16$ & $1.7 \pm 0.16$ & $65.0 \pm 9.35$ & $6.9 \pm 0.49$ & $45.0 \pm 3.7$ \\
\hline & $P$ & $<0.05$ & $<0.01$ & $<0.01$ & $<0.05$ & $<0.01$ \\
\hline Control group & & $1.8 \pm 0.17$ & $1.14 \pm 0.03$ & 0 & $5.2 \pm 0.71$ & $28.8 \pm 4.48$ \\
\hline
\end{tabular}

$P$ - vs. control group 
Table 2.

Concentration (mg/100ml) of Phe and Tyr in the blood serum of VH patients with the severe form of disease after Phe load

\begin{tabular}{|lc|c|c|c|c|}
\hline \multirow{2}{*}{$\begin{array}{c}\text { Time after } \\
\text { Phe load } \\
\text { (hours) }\end{array}$} & \multicolumn{2}{|c|}{ Control } & \multicolumn{2}{c|}{ Patients } \\
\cline { 3 - 6 } & & $1.8 \pm 0.17$ & $1.14 \pm 0.003$ & $3.86 \pm 0.65$ & $1.97 \pm 0.193$ \\
& $P$ & & & $<0.01$ & $<0.01$ \\
1 & & $10.7 \pm 0.88$ & $1.53 \pm 0.12$ & $10.35 \pm 1.05$ & $2.08 \pm 0.19$ \\
& $P$ & & & $<0.8$ & $<0.02$ \\
2 & & $6.6 \pm 1.66$ & $1.61 \pm 0.12$ & $9.52 \pm 0.80$ & $2.14 \pm 0.22$ \\
& & & & $<0.2$ & $<0.05$ \\
4 & & $2.2 \pm 0.17$ & $1.65 \pm 0.29$ & $8.4 \pm 0.86$ & $2.17 \pm 0.18$ \\
& & & & $<0.01$ & $<0.02$ \\
6 & & $1.7 \pm 0.15$ & $1.51 \pm 0.13$ & $7.1 \pm 0.67$ & $2.23 \pm 0.15$ \\
& & & & $<0.01$ & $<0.01$ \\
\hline
\end{tabular}

Table 3.

Liver PAH activity ( $\mu$ mol of Tyr/g protein/hour), the levels of Phe and Tyr in the blood serum (mg/100 $\mathrm{ml})$, and PPA excretion in daily urine $(\mathrm{mg} / 100 \mathrm{ml})$ in $\mathrm{VH}$ patients

\begin{tabular}{|c|c|c|c|c|c|c|c|c|}
\hline Patients & Age & $\begin{array}{l}\text { Severity } \\
\text { of disease }\end{array}$ & $\begin{array}{l}\text { Stage of } \\
\text { disease }\end{array}$ & Phe & Tyr & PPA & $\begin{array}{r}\text { Liver } \\
\text { acti } \\
+ \text { cofactor }\end{array}$ & $\begin{array}{l}\text { r PAH } \\
\text { ivity } \\
\text { r.-cofactor }\end{array}$ \\
\hline 1. S.V. & 42 & Mild & & 2.0 & 0.89 & 0.2 & 55.2 & - \\
\hline 2. C.G. & 47 & Moderate & Peak & 3.1 & 1.52 & 5.3 & 23.6 & 2.6 \\
\hline 3. S.E. & 67 & Moderate & Peak & 3.5 & 2.4 & 2.8 & 18.8 & 2.0 \\
\hline 4. S.A. & 26 & Mild & Regression & 2.8 & 1.16 & 1.3 & 50.0 & - \\
\hline 5. P.V. & 44 & Moderate & Peak & 3.0 & 1.23 & 0.5 & 30.6 & - \\
\hline
\end{tabular}

Investigating $\mathrm{PAH}$ activity, we established that the activity of the enzyme makes $0.7 \%$ to $13 \%$ and even less to total absence in PKU patients [22], 43\% for heterozygous carriers [23], and $44 \%$ for $\mathrm{VH}$ patients in comparison with data for people without any liver pathology.

Comparing data of Phe load with data for PAH activity in livers of $\mathrm{VH}$ patients; it is possible to conclude that the disturbance in reaction of Phe hydroxylation with $\mathrm{VH}$ is caused by the decrease in PAH activity in the liver. Inasmuch as PAH activity is normalized ambiguously with various forms of $\mathrm{VH}$ during the recovery period, this activity can serve as one of the indicators of the existence and intensity the pathological process, as well as the efficiency of treatment.

The insufficiency of liver function in AAA metabolism may be considered as the main factor among the major factors responsible for $\mathrm{HE}[5,8]$, which causes an imbalance of biogenic amines that, penetrating through the hematoencephalic barrier, disturb the function of neurons. This disturbance leads to the emergence of mental and neurologic disorders [8].

The study of catecholamine metabolism with liver disease showed the role of "false mediators" in the development of hepatic insufficiency. It is believed that impairment of consciousness among patients with hepatic insufficiency is associated with a reduction in the formation of neurochemical transmitters, such as norepinephrine and dopamine. Studies of monoamines in the brains of patients who died from hepatic coma have revealed a decrease in the level of dopamine and an increase in the concentration of serotonin in all areas of brain [8].

With $\mathrm{VH}$, an increase in the activity of bacterial amino acid decarboxylases causes the formation of substances like tyramine and octopamine with a structure similar to norepinephrine; consequently, these substances can collect in the nervous system, replacing norepinephrine. These false mediators disturb the neurochemical transfer in the brain and cause the HE syndrome. The introduction of DOPA, which is the predecessor of norepinephrine and easily penetrates into the brain, causes a strengthened formation of norepinephrine in the brain and replaces the false mediators.

The accumulation of substances relating to phenolic compounds plays an important role in AHF pathogenesis. It has been found that a toxic effect is produced by free phenol and AAA, as well as by their metabolites - the phenolic compounds that were increased in the blood, cerebrospinal fluid, and urine of patients with the severe forms of $\mathrm{VH}$ and hepatic coma [8].

Direct determination of PAH activity in liver bioptates of VH patients has shown a decrease in enzyme activity. Currently, more than 500 various mutations in the PAH gene have been discovered, and the frequency of the mutant alleles is 1:50 in the general population [24]. The frequency of complications of AHF with hepatic coma is $1: 40$ to $1: 100$ in patients. It is quite probable that $\mathrm{AHF}$ and coma with $\mathrm{VH}$ develop in patients who are heterozygous for $\mathrm{PAH}$ gene mutation, while the homozygous patients for the wild-type gene undergo $\mathrm{VH}$ in mild forms or act as virus carriers.

All these data were typical for our patients. The severity of psychoneurological symptoms (emotional instability, dizziness, the slow thinking, disorientation, tremors of hands, the confused consciousness, and psychomotor excitement) was correlated with the severity of phenylalanine and tyrosine metabolism disorders, and the severity decreased with normalization of these indicators.

Thus, the data we obtained confirm disturbances of AAA metabolism and a decrease in PAH activity by $44 \%$ in $\mathrm{VH}$ patients with the moderate-to-severe and severe forms of disease. These data also reveal disturbances of hydroxylation of Phe to Tyr. PAH-system dysfunction is the trigger for development of Tyr and Trp metabolism disorders that are accompanied by HE during development of AHF. Inasmuch as PAH activity is normalized ambiguously with various forms of $\mathrm{VH}$ during the recovery period, this activity can serve as one of the indicators of the existence and intensity the pathological process, as well as the efficiency of treatment. In this state, the inclusion of tetrahydrobiopterin in the treatment scheme is appropriate since it leads to the normalization of AAA metabolism.

Table 4 shows the serum levels of Phe and Tyr and their metabolites in urine of patients suffering from alcoholism and in those of the control group. It can be seen that, in comparison with the control group, patients suffering from alcoholism had an increased serum Phe level, while the Tyr level was decreased.

The changes in Phe/Tyr ratio are especially vivid. The most expressed disturbances in the content of blood amino 
acids were revealed in patients with the abstinence syndrome. As for the patients in the remission period, despite their abstention from alcohol for a month or more, when practically all clinical indicators are normalized, the Phe level remained elevated, while Tyr content was decreased, in comparison with the control group. The urine test showed that the Phe and Tyr metabolism of patients with alcoholism was drastically changed. Disturbances of Phe metabolism in patients with the alcoholic abstinence syndrome lead to a pathological increase in PPA excretion, which is absent in the urine of healthy people and patients with alcoholism in the remission stage. The excessive urinary excretion of Phe was detected both in the abstinence syndrome and in the remission stage. Decreased excretion of HGA, a metabolite of Tyr, indicates the disturbances in the metabolism of Tyr.

\section{Table 4.}

Serum levels of Phe and Tyr, Phe/Tyr ratio and excretion of Phe, $P P A$ and $H G A$ in daily urine of patients with chronic alcoholism $(\mathrm{mg} / 100 \mathrm{ml})$

\begin{tabular}{|l|c|c|c|c|}
\hline \multirow{2}{*}{$\begin{array}{c}\text { Study } \\
\text { samples }\end{array}$} & \multirow{2}{*}{ Indicator } & \multicolumn{2}{|c|}{ Patients with chronic alcoholism } & \multirow{2}{*}{ Control } \\
\cline { 3 - 5 } & & $\begin{array}{c}\text { abstinence } \\
\text { syndrome }\end{array}$ & $\begin{array}{c}\text { remission } \\
\text { stage }\end{array}$ & \\
\hline \multirow{3}{*}{ Serum } & Phe & $2.43 \pm 0.16^{*}$ & $1.96 \pm 0.05$ & $1.80 \pm 0.17$ \\
& Tyr & $0.96 \pm 0.09^{*}$ & $0.83 \pm 0.018^{*}$ & $1.14 \pm 0.03$ \\
& Phe/Tyr & 2.53 & 2.36 & 1.58 \\
\hline \multirow{3}{*}{ Urine } & PPA & $5.7 \pm 0.6^{*}$ & 0 & 0 \\
& HGA & $8.2 \pm 0.6^{*}$ & $8.6 \pm 1.47$ & $10.4 \pm 0.03$ \\
& Phe & $2.83 \pm 0.16^{*}$ & $2.1 \pm 0.11$ & $1.9 \pm 0.23$ \\
\hline
\end{tabular}

$* P<0.01$ vs. control

The disorders in Phe metabolism found with alcoholism are similar to the disorders with $\mathrm{VH}$, and also to the results received at Phe load in heterozygous carriers for the R408W mutation of the PAH gene, which is associated with genetically determined reduction in PAH activity (PAH activity was less than $50 \%$ in comparison with the healthy people).

Especially pronounced metabolic disorders in Phe and Tyr metabolism have been found in patients with abstinence syndrome. In these patients, serum Phe concentration was much higher, and the level of Tyr was lower, in comparison with the control group. The urinary excretion of PPA was increased, and the HGA excretion was decreased, which showed the disturbance of Tyr metabolism with chronic alcoholism.

The data obtained allow us to assume that there is an interrelation between the severity of mental disturbances associated with alcoholic intoxication and the severity of PAHsystem disorders as well as Phe and Tyr metabolism distortion. The decrease in the activity of the liver PAH-system can lead to accumulation of Phe and its pathological derivatives in the brain tissues, which causes disorders of biogenic amine metabolism in CNS.

We also assumed that patients with CA, especially those who had delirium tremens in the anamnesis, may have mutations in the PAH gene, ie, these patients may be heterozygous carriers of the R408W mutation. For this purpose, the group of patients with alcoholism (stages 2 and 3) was tested for R408W mutation typical for PKU.
According to several studies, the average frequency of the heterozygous carriage of PKU in various populations is $1: 40$ to $1: 200$. When we examined the control group for the $\mathrm{R} 408 \mathrm{~W}$ mutation, which is typical for $65 \%$ to $70 \%$ of PKU patients, our experimental data have been consistent with data of other researchers; ie, the frequency of PKU heterozygous carriage of the R408W mutation was $2.39 \%$.

In patients with $\mathrm{CA}$, the frequency of heterozygous carriage of the R408W mutation was much higher: Among 156 patients with CA, 11 people had a heterozygous state of $7.05 \%(P<0.02)$.

Because the number of PKU heterozygous carriers was clearly higher among patients with CA, we assume that the disturbance of the hepatic PAH activity contributes to the development of alcoholic illness and delirium tremens. One of the possible mechanisms for such a phenomenon is the congenital disorder in metabolism of CNS neurotransmitters in PKU heterozygous carriers, which manifests in psychological discomfort, emotional instability, and a tendency toward deviant behavior. Alcohol intake, on the one hand, temporarily softens such symptomology, and on the other, accelerates the process of alcoholization and the phenomena of mental and physical dependence, and leads further to a heavier somatic and psychoneurological frustration in this group of patients.

\section{Competing interests}

The authors declare that they have no competing interests.

\section{References}

1. Brumm VL, Bilder D, Waisbren SE. Psychiatric symptoms and disorders in phenylketonuria. Mol Genet Metab 2010; 99 Suppl 1:S59-63.

2. Huttenlocher PR. The neuropathology of phenylketonuria: human and animal studies. Eur J Pediatr 2000; 159 Suppl 2:S102-6.

3. Moyle JJ, Fox AM, Arthur M, Bynevelt M, Burnett JR. Meta-analysis of neuropsychological symptoms of adolescents and adults with PKU. Neuropsychol Rev 2007; 17(2):91-101. 4. Perez-Duenas B, Pujol J, Soriano-Mas C, Ortiz H, Artuch $\mathrm{R}$, Vilaseca MA, et al. Global and regional volume changes in the brains of patients with phenylketonuria. Neurology 2006; 66(7):1074-8.

5. Cash WJ, McConville P, McDermott E, McCormick PA, Callender ME, McDougall NI. Current concepts in the assessment and treatment of hepatic encephalopathy. Q J Med 2010; 103(1):9-16.

6. Dejong $\mathrm{CH}$, van de Poll MC, Soeters PB, Jalan R, Olde Damink SW. Aromatic amino acid metabolism during liver failure. J Nutr 2007;137(6 Suppl 1):1579S-1585S.

7. Toris GT, Bikis CN, Tsourouflis GS, Theocharis SE. Hepatic encephalopathy: an updated approach from pathogenesis to treatment. Med Sci Monit 2011; 17(2):RA53-63.

8. Ivashkin VT. Liver and Biliary System Diseases. Manual for doctors. M.: Publishing house «M-Vesti», 2005. [Manual in Russian].

9. Maier KP. Hepatitis - Hepatitisfolgen. Praxis der Diagnostik, Therapie und Prophylaxe akuter und chronischer Lebererkrankungen. Hans Huber Verlag, 6. Aufl. 2010. [Book in German]. 
10. Shaposhnikov AM, Khal'chitskiı̌ SE, Shvarts EI. Disorders of phenylalanine and tyrosine metabolism in Down's syndrome. Vopr Med Khim 1979; 25(1):15-9.[Article in Russian]

11. Dooley JS, Lok A, Burroughs AK, Heathcote J, editors. Sherlock's Diseases of the Liver and Biliary System. WileyBlackwell 12th Edition; 2011.

12. Hörtnagl H, Lochs H, Kleinberger G, Hackl JM, Hammerle AF, Binder $\mathrm{H}$, Wewalka F. Plasma catecholamines in hepatic coma and liver cirrhosis: role of octopamine. Klin Wochenschr 1981; 59(20):1159-64.

13. Krauns P, Ruge W. Plasma catecholamine levels in liver disease. Z Gastroenterol 1985; 23(2):64-73. [Article in German].

14. Mukherjee S, Das SK, Vaidyanathan K, Vasudevan DM. Consequences of alcohol consumption on neurotransmitters an overview. Curr Neurovasc Res 2008; 5(4):266-72.

15. Sherlock S. Alcoholic liver disease. Lancet 1995; 345(8944):227-9.

16. Ehomoto N., Takase S., Takada N, Takada A. Alcoholic liver disease in heterozygotes of mutant and normal aldehyde dehydrogenase-2 genes. Hepatology 1991; 13(6):1071-5.

17. Shaposhnikov AM, Khal'chitskiı̌ S.E. Patochemistry of phenylalanine, tyrosine, tryptophan metabolism and activity of liver phenylalanine hydroxylase in viral hepatitis. Natur Tech Science 2007; 2: 137-154 [Article in Russian].
18. Mukherjee S, Vaidyanathan K, Vasudevan DM, Das SK. Role of plasma amino acids and GABA in alcoholic and non-alcoholic fatty liver disease-a pilot study. Indian J Clin Biochem 2010; 25(1):37-42.

19. Bulycheva I.A., Khalchitsky S.E. Diagnostic importance of phenylpyruvic acid definition in urine in alcoholic disease. Medical sciences 2010; 2:40-49 [Article in Russian].

20. Gofman AG. Clinical Narcology. Moscow: Miclosh, 2003, 23-34 [Book in Russian].

21. Westwood A and Raine DN. Heterozygote detection in phenylketonuria. Measurement of discriminatory ability and interpretation of the phenylalanine loading test by determination of the heterozygote likelihood ratio. J Med Genet 1975; 12(4):327-333.

22. Shaposhnikov AM, Barashnev IuI, Khal'chitskiū SE, Korneǐchuk VV, Okat'ev VS. Phenylalanine hydroxylase activity in the liver in children with the classic form of phenylketonuria._Vopr Okhr Materin Det 1978;23(6):42-7. [Article in Russian]

23. Shaposhnikov AM. Biochemical manifestations of genetic heterogeneity in human hereditary enzymopathy. Diss Doctor Med Sci. Leningrad, 1975. [Dissertation in Russian].

24. Williams RA, Mamotte CD, Burnett JR. Phenylketonuria: an inborn error of phenylalanine metabolism. Clin Biochem Rev 2008; 29(1):31-41. 